\title{
Avaliação do emprego da técnica de análise de regressão logística em teses e dissertações de algumas instituições de ensino superior
}

\section{Evaluation of the use of the logistic regression analysis technique on theses and dissertations of some higher education institutions}

\author{
Leandro Campi Prearo ${ }^{1}$, Maria Aparecida Gouvêa ${ }^{2}$, Carolina Monari ${ }^{3}$
}

\begin{abstract}
Resumo
Este artigo faz parte de um amplo estudo de avaliação da adequação no uso de técnicas estatísticas multivariadas na área de marketing, em teses e dissertações acerca do do comportamento do consumidor entre 1997 e 2006. Neste artigo, é focalizada a análise de regressão logística, uma técnica com grande potencial de uso em estudos de marketing. O objetivo deste trabalho foi a análise da adequação do emprego dessa técnica às necessidades dos problemas de pesquisa apresentados nas teses e dissertações e a aferição do nível de acerto no atendimento de suas premissas. De forma geral, os resultados sugerem a necessidade de um aumento do comprometimento dos pesquisadores na verificação de todos os preceitos teóricos de aplicação da técnica de análise de regressão logística.

Palavras-chave: Análise multivariada. Regressão logística. Comportamento do consumidor.
\end{abstract}

\begin{abstract}
This paper is part of a comprehensive study to assess the suitability of the use of multivariate statistical techniques in theses and dissertations of some higher education institutions in the marketing area on the topic of consumer behavior between 1997 and 2006. In this article the logistic regression analysis is focused, a technique with great potential for use in marketing studies. This study aimed at analyzing the suitability of this technique to the needs of research problems presented in the theses and dissertations and measuring the level of success in meeting their premises. Overall, the results suggest the need for an increased commitment of researchers to verify all theoretical rules of application of the logistic regression analysis technique.
\end{abstract}

Key words: Multivariate analysis. Logistic regression. Consumer behavior.

\section{Introdução}

A qualidade da produção científica nacional em Administração tem recebido a atenção de muitos acadêmicos, sobretudo a partir da década de 1990: Administração Pública (MACHADODA-SILVA; CUNHA; AMBONI, 1989); Finanças
(LEAL; OLIVEIRA; SOLURI, 2003); Marketing (POWERS et al., 1998; VIEIRA, 1998; BOTELHO; MACERA, 2001); Métodos Quantitativos (BREI; LIBERALI, 2004); Operações (ARKADER, 2003), Organizações (MACHADO-DA-SILVA; CUNHA; AMBONI, 1990; BERTERO; KEINERT, 1994;

\footnotetext{
1 Mestre em Administração - Área de Métodos Quantitativos - FEA/USP. Docente e pesquisador da Universidade Municipal de São Caetano do Sul (USCS) São Caetano do Sul - São Paulo. Rua Engenheiro Luiz La Scala Junior, 40 - AP 54 - Bairro Rudge Ramos São Bernardo do Campo - São Paulo - CEP 09625-020.

2 Livre-Docência em Administração - Faculdade de Economia, Administração e Contabilidade da Universidade de São Paulo - FEA/USP. Docente de Estatística e Metodologia de Pesquisa - Curso de Graduação e Pós-Graduação da FEA/USP no Departamento de Administração da FEA/USP. E-mail: magouvea@usp.br. Endereço: Av. Prof. Luciano Gualberto, 908 - FEA 1 - Sala E110 - Cidade Universitária - CEP 05508-900 - São Paulo- São Paulo.

3 Aluna do Curso de Graduação em Administração - Faculdade de Economia, Administração e Contabilidade da Universidade de São Paulo - USP. E-mail: cmonari@gmail.com. Endereço: Av. Prof. Luciano Gualberto, 908 - FEA 1 - Sala E110 - Cidade Universitária - CEP 05508-900 - São Paulo.
} 
VERGARA; CARVALHO， 1995; BERTERO; CALDAS; WOOD JUNIOR, 1999; RODRIGUES FILHO, 2002), Pesquisa em Administração (MARTINS, 1994; TORRES, 2000; PERIN et al., 2002); Recursos Humanos (ROESCH; ANTUNES, SILVA, 1997; CALDAS; TONELLI; LACOMBE, 2002; TONELLI et al., 2003), Sistemas de Informação (HOPPEN et al., 1998).

Em geral, essa análise crítica da produção acadêmica nacional se divide em duas linhas: a linha das discussões acerca dos aspectos epistemológicos e a linha cuja ênfase recai na adoção de critérios de qualidade e consistência da produção em Administração, ou seja, nos aspectos metodológicos. Essa segunda linha parece ainda contar com um número reduzido de estudos.

Martins (1994, p. 65-66), evidenciou, em estudo sobre a Epistemologia da Pesquisa em Administração, que as dissertações e teses apresentadas na FEA/USP, FEA/PUC e EASP/ FGV, entre os anos de 1980 e 1993, abusavam da utilização do instrumental dos métodos quantitativos de forma bastante superficial, dado o nível de sofisticação dessas técnicas. $\mathrm{O}$ autor alertou ainda para a despreocupação para com as premissas teóricas de aplicação da maior parte dos métodos.

Para Gamboa (1987, p. 17), a importância da análise da produção científica, amparada nas competências metodológicas, justifica-se por tratar-se de uma questão de fundamental e decisiva importância para o desenvolvimento e resultados da pesquisa.

Aevidentecontribuiçãoqueaanálisemultivariada pode oferecer à análise quantitativa dos dados para a produção científica alia-se ao fato de que, nas últimas décadas, vários pacotes computacionais estatísticos se aperfeiçoaram sobremaneira, com o objetivo de tornar seus conteúdos distantes das complexidades matemáticas, próprias dos mesmos.

As técnicas de análise multivariada têm sofrido sofisticações para atender a demanda dos cientistas das ciências sociais aplicadas, entre elas da Administração. Essas sofisticações das ferramentas de análise atraem os pesquisadores pelas facilidades oferecidas, mas podem levá-los a erros de aplicação, seja na inadequação dos objetivos de uso das ferramentas com os objetivos propostos na pesquisa, seja na violação de premissas de aplicação das técnicas. Há situações em que o pesquisador apenas exercita o emprego de uma técnica e distancia-se de seu problema de pesquisa e do alcance dos objetivos inicialmente traçados.

Uma das áreas da Administração que mais utiliza o método quantitativo, especialmente a análise multivariada, é a área de Marketing, e isso ocorre sobretudo pela necessidade de se conhecer o mercado consumidor busca-se a mensuração das opiniões, atitudes, preferências, perfil e outras características dos consumidores (MALHOTRA, 2001).

Os estudos em Marketing, especialmente na temática do Comportamento do Consumidor, utilizam-se com frequência das ferramentas de análise multivariada. Para Milagre (2001, p. 74), o uso da técnica multivariada tornouse mais comum a partir do momento em que os acadêmicos e profissionais de Marketing passaram a aplicá-la em estudos a respeito da preferência e satisfação do consumidor, bem como do seu perfil e comportamento de compras. Martins (1994, p. 66) afirma que, em Administração, os métodos quantitativos são mais comumente aplicados pelos autores de pesquisa nas áreas de Marketing, Produção e Finanças.

Nesse sentido, neste estudo pretende-se aprofundar a discussão e oferecer subsídios à reflexão na temática dos Métodos Quantitativos, especialmente sob o recorte de suas aplicações nos estudos em Marketing sobre o Comportamento do Consumidor.

A análise de regressão logística (logistic regression analysis) é uma técnica com grande potencial de aplicação na área de Marketing, 
sobretudo pelo fato de possibilitar a caracterização de grupos definidos de acordo com alguma variável de interesse na pesquisa (por exemplo, grupo de leais versus grupo de não leais a uma marca) ou gerados após a aplicação da análise de conglomerados. A análise de regressão logística é também empregada na atividade de segmentação de mercado, um dos principais alicerces para a compreensão do comportamento do consumidor.

Neste contexto, os objetivos a serem alcançados neste estudo são:

A. Identificar a intensidade de uso das técnicas multivariadas na área de Marketing, temática do Comportamento do Consumidor, tendo como unidade de análise dissertações e teses, do período 1997-2006, de duas universidades públicas: Faculdade de Economia, Administração e Contabilidade da Universidade de São Paulo e Escola de Administração da Universidade Federal do Rio Grande do Sul.

B. Identificar o nível de adequação do uso da técnica de análise de regressão logística aos problemas de pesquisa apresentados nestas dissertações e teses selecionadas.

C. Identificar fontes de erros da aplicação da técnica de análise de regressão logística, a partir do não atendimento às suas premissas básicas, no conjunto da produção científica examinada.

A seleção destes Programas de Pós-Graduação como público-alvo desse estudo deveu-se à necessidade de delimitação, dada a dificuldade operacional de um levantamento amostral representativo da produção nacional, principalmente pela indisponibilidade de material em base de dados on-line por uma parcela importante das Instituições do país.

Nesse sentido, optou-se inicialmente por um recorte focado nos Programas de Pós-Graduação de alta performance na última avaliação da Coordenação de Aperfeiçoamento de Pessoal de Nível Superior (CAPES). Registre-se que a opção por esse critério não se motivou pela suposição de que os programas com avaliação menos positiva, abaixo da nota 6 , tratariam as técnicas em estudo de forma mais ou menos corretas. A avaliação da CAPES aponta três Instituições com nota 6: a FEA/ USP, a EA/UFRGS e a EASP/FGV.

Um segundo critério deu-se da necessidade de garantir a maior homogeneidade possível da população alvo, já que não é objetivo específico desse estudo a comparação entre Instituições diferentes. Nesse sentido, a seleção levou em conta a natureza da Instituição: pública ou privada. Considerando que entre as três citadas, duas são públicas, optou-se pela análise das dissertações e teses das Instituições públicas: FEA/USP e EA/ UFRGS.

Mencionam-se a seguir alguns fatores restritivos na abrangência dessa investigação:

- Delimitação do público alvo: os resultados desse estudo são apenas válidos para as dissertações e teses da FEA/USP e da EA/ UFRGS, de 1997 a 2006.

- As informações quanto ao atendimento às premissas da análise de regressão logística são baseadas nos relatos dos autores de cada dissertação ou tese sob análise, que, evidentemente, podem conter uma descrição subestimada ou superestimada do que foi realizado.

\section{Fundamentação Teórica}

Nesta seção, haverá uma introdução acerca da análise multivariada, seguida por uma apresentação geral de técnicas multivariadas, finalizando com considerações sobre a análise de regressão logística. 


\section{Análise multivariada}

A análise multivariada permite estudar e evidenciar as ligações, as semelhanças e as diferenças existentes entre todas as variáveis envolvidas no processo (BOUROCHE; SAPORTA apud TRIVELLONI; HOCHEIM, 2001). Segundo Steiner (1995), a necessidade de entender a relação entre diversas variáveis aleatórias faz da análise multivariada uma metodologia com grande potencial de uso. Para Lourenço e Matias (2001), por um lado, as técnicas estatísticas multivariadas são mais complexas do que aquelas da estatística univariada. Por outro lado, apesar de uma razoável complexidade teórica fundamentada na matemática, as técnicas multivariadas permitem o tratamento de diversas variáveis ao mesmo tempo, e, assim, podem oferecer ao pesquisador um material bastante consistente para a análise dos dados da pesquisa.

Conforme Hair et al. (2005), a análise multivariada auxilia a formulação de questões relativamente complexas de forma específica e precisa, e isso possibilita a condução de pesquisas teoricamente significativas.

\section{Técnicas estatísticas de análise multivariada}

A escolha dos métodos e tipos de análises empregadas nos trabalhos científicos deve ser determinada pelo problema de pesquisa. Nesse sentido, Johnson e Wichern $(1998$, p. 2) propõem uma classificação dos objetivos para atendimento do problema em cinco categorias.

\section{Classificação \\ Investigação da dependência entre as variáveis}

Todas as variáveis são mutuamente independentes, ou uma ou mais variáveis são dependentes de outras.

\section{Técnicas relacionadas}

Análise Discriminante

Análise de RegressãoC orrelação Canônica

Regressão Logística

Análise Conjunta

MANOVA

Análise Discriminante

Análise de Regressão

Análise de Regressão Logística

Hipóteses estatísticas específicas, formuladas em termos de parâmetros da população multivariada, são testadas. Isso pode ser feito para validar premissas ou para reforçar convicções prévias.

\section{Redução dos dados ou simplificação estrutural}

O fenômeno em estudo é representado de um modo tão simples quanto possível, sem sacrificar informações importantes.

\section{Redução dos dados ou simplificação estrutural}

O fenômeno em estudo é representado de um modo tão simples quanto possível, sem sacrificar informações importantes.

\section{Agrupamento de objetos ou variáveis}

Grupos de objetivos ou variáveis "similares" são criados com base nas medidas características.
Modelagem de Equações Estruturais

Análise Fatorial Confirmatória

\section{Análise Fatorial Exploratória}

Análise de Conglomerados

Análise de Correspondência

Escalonamento Multidimensional 
Após considerar os objetivos do problema de pesquisa, o passo seguin te para a escolha da técnica de análise multivariada é verificar o tipo de relação examinada, número de variáveis dependentes e tipo de escala utilizada.

Sobreotipoderelação, astécnicassãoclassificadas como de dependência ou de interdependência. $\mathrm{Na}$ primeira situação, uma ou mais variáveis (variáveis dependentes) podem ser explicadas ou preditas por outras (variáveis independentes). Na segunda, todas as variáveis são analisadas simultaneamente, sem a orientação de dependência ou independência.

Quanto ao tipo de escala utilizada, pode-se generalizar a classificação teórica dessas escalas de mensuração em dois grandes grupos: variáveis métricas e variáveis não métricas.

\section{Análise de regressão logística}

Segundo Cramer (2002), os primeiros estudos que levaram ao atual estágio de aplicação da regressão logística foram desenvolvidos no século XIX para descrever o crescimento das populações.

Em artigo publicado em 1845 na revista "Proceedings", da Belgian Royal Academy, PierreFrançois Verhulst (1804-1849) definiu uma função para tratar do crescimento exponencial da população e a nomeou função logística, devido ao diagrama da curva ser parecido com a curva logarítmica, atualmente denominada de exponencial.

Não obstante, a função logística só foi reconhecida pelo mundo acadêmico-científico em 1920, a partir de estudo desenvolvido por Pearls e Reed a respeito do crescimento da população norteamericana.

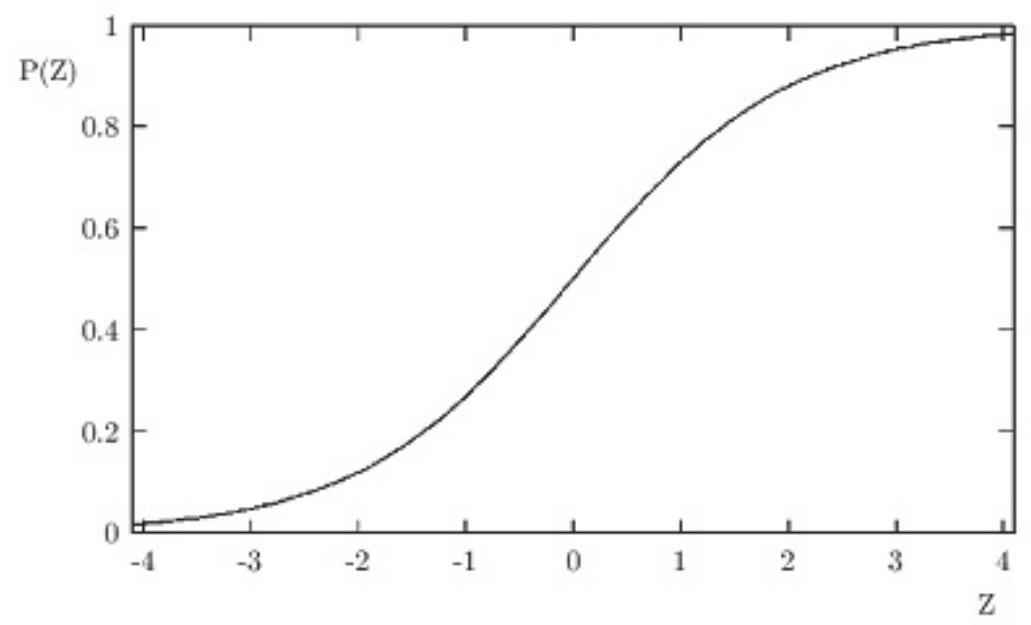

Figura 1. A curva logística

Fonte: Cramer (2002).

No tocante aos objetivos de aplicação, a análise de regressão logística enquadra-se na categoria de investigação da dependência entre as variáveis e na categoria predição 
Descrever a relação entre a ocorrência ou não de evento de interesse, a partir de um conjunto de variáveis explanatórias.

Frees (1996)

- Investigação da dependência entre as variáveis.

- $\quad$ Predição
Identificar se as diferenças estatísticas existem entre as pontuações de um conjunto de variáveis para dois ou mais grupos definidos anteriormente.

\section{Predizer uma variável dependente} a partir de um grupo de variáveis independentes, e determinar o quanto a variável dependente foi explicada por esse grupo.
Hair et al. (2005)

\section{Garson (2007c)}

Quadro 2. Objetivos de aplicação da técnica de Análise de Regressão Logística

\section{Premissas da técnica estatística de análise de regressão Logística}

Em função da naturesza não linear da função logística, a técnica Regressão Logística Binária utiliza para a estimação dos parametros o método de Máxima Verossimilhança. Sob a ótica das suposições que geralmente permeiam a utilização de técnicas de dependência, como a normalidade multivariada e a homocedasticidade, a grande importância desse método de estimação reside em ele requerer bem menos suposições que, por exemplo, a Análise Discriminante ou Regressão Múltipla e, ainda assim, apresentar boas propriedades assintóticas dos estimadores, que são consistentes e assintoticamente eficientes.

A seguir, são comentadas as premissas presentes na análise de regressão logística.

\section{Multicolinearidade}

Segundo Gujarati (2000, p. 318), o termo multicolinearidade foi cunhado por Ragnar Frish em 1934. Originalmente, significa a existência de uma "perfeita" (ou exata) relação linear entre algumas ou todas as variáveis explicativas de um modelo de regressão. Formalmente, o termo refere-se à existência de mais de uma relação linear exata, ao passo que o termo colinearidade refere-se à existência de uma única relação linear.

Algumas sugestões para o tratamento de multicolinearidade, a partir da leitura de Tabachnick e Fidell (1996), Gujarati (2000), Pestana e Gageiro (2000), Hair et al. (2005), Garson (2007a): Ignorar se o objetivo é prever.

- Eliminar variáveis.

- Reformular o modelo - usando, por exemplo, a razão entre variáveis.

- Aumentar o tamanho da amostra.

A literatura consultada apresenta uma série de testes estatísticos para avaliação da multicolinearidade, entre os quais se mencionam os de Hair et al. (2005) e Garson (2007c):

- $\quad$ MSA (Measure of Sampling Adequacy)

- $\quad$ KMO (Kaiser-Meyer-Olkin)

- VIF (Variance inflation factor)

- Índice de condição 
- Índice de tolerância

Influência de dados extremos univariados e multivariados

As observações atípicas ou extremas (outliers) são aquelas substancialmente diferentes das outras. Podem ser identificadas no nível univariado, bivariado ou multivariado.

Segundo Hair et al. (2005) e Garson (2007a), alguns fatores podem causar a presença de observações atípicas univariadas nos dados coletados, e alterar o resultado de análise e, também, causar violações de normalidade:

Erro na entrada de dados ou na codificação.

- Evento extraordinário: o pesquisador deve decidir se cabe ou não manter o dado extremo na análise.

- Observações extraordinárias para os quais o pesquisador não tem explicação.

- Observações que representam uma combinação única de valores das variáveis: o pesquisador deve manter o dado a menos que haja evidência específica para desconsiderá-lo.

Os outliers multivariados consistem em casos com valores extremos no conjunto de múltiplas variáveis e são operacionalmente definidos por meio de três medidas mais comumente usadas e disponíveis na maioria dos pacotes estatísticos comerciais: a Distância de Cook, a Distância de Mahalanobis e a Distância de Leverage.

\section{Sensibilidade ao tamanho da amostra}

Aliado a um correto procedimento de amostragem, o tamanho da amostra (número de casos, indivíduos, observações, entrevistas) deve ser adequado para permitir a generalização dos resultados, os quais podem ser verificados quanto à significância estatística dos testes.

As facilidades oferecidas pela maioria dos pacotes computacionais de aplicação estatística pode comprometer a observância do tamanho mínimo da amostra pelo pesquisador, e esse fato pode causar uma série de problemas importantes. Para Hair et al. (2005), essa omissão pode resultar em:

- baixíssimo poder estatístico dos testes de significância;

- um ajuste muito adequado dos dados, tornando os resultados artificialmente bons.

Segundo Garson (2007c), a Regressão Logística é em parte mais utilizada, porque permite ao pesquisador superar muitas suposições restritivas presentes na Análise de Regressão e na Análise Discriminante.

Assim, a Regressão Logística não assume linearidade das relações, nem que o erro seja normalmente distribuído, e também não faz exigência quanto à normalidade da distribuição multivariada. Ela assume, porém que a distribuição esteja dentro da gama da família exponencial das distribuições, como Normal, Poisson, Binomial e Gama), além de também relaxar a premissa de homoscedasticidade.

O Quadro 2 sintetiza as premissas da técnica de análise de regressão logística. 
Multicolinearidade (2)

Ausência de observações atípicas(2)

Tamanho da Amostra (1) (2)

10 casos para cada variável integrante do modelo(1)

Quadro 3. Premissas subjacentes ao uso da Análise de Regressão Logística

Fonte: Elaboração própria a partir da abordagem de (1) Peduzzi et al (1996); (2) Garson (2007c)

\section{Metodologia de Pesquisa}

Nessa seção, serão apresentados os tópicos referentes aos seguintes itens : a caracterização da população, o desenho metodológico da pesquisa empírica, a construção do critério de adequação da aplicação da técnica de análise de regressão logística, instrumento de coleta de dados e variáveis de resultados.

\section{Caracterização da população-alvo}

Retomando as considerações iniciais acerca do público-alvo, a análise contempla as dissertações e teses em Administração, área de Marketing, temática do Comportamento do Consumidor, apresentadas nos Programas de Pós-Graduação da Faculdade de Economia, Administração e Contabilidade da Universidade de São Paulo e da Escola de Administração da Universidade Federal do Rio Grande do Sul, no período entre 1997 e 2006.

O universo de interesse foi definido como a dissertação ou tese, com foco em Marketing, especificamente comportamento do consumidor, e utilização de técnica estatística de análise multivariada como instrumento de solução do problema de pesquisa, apresentada aos PPGA's das instituições públicas com mais alta avaliação pela CAPES, na área de Administração.

A unidade populacional, no entanto, refere-se à aplicação de técnica estatística multivariada nesse universo de interesse, e pode ser mais do que uma aplicação em cada estudo integrante do universo de interesse.

A identificação da população-alvo foi realizada em três etapas:

- Seleção dos trabalhos que apresentavam o termo comportamento do consumidor como uma das palavras-chave.

- Leitura dos resumos dos trabalhos não selecionados na primeira etapa, com o objetivo de identificar aqueles que tratavam do comportamento do consumidor, ainda que não apresentassem o termo como palavra-chave, e seleção dos trabalhos pertinentes.

- Exame do conteúdo dos trabalhos selecionados na primeira e na segunda etapa, identificando aqueles com aplicação de alguma técnica de análise multivariada.

A Tabela 1 apresenta o ambiente de identificação da população-alvo desse estudo, registrando 196 dissertações e teses acerca de Comportamento do Consumidor (universo - U), 56 dissertações e teses com aplicação de técnicas estatísticas multivariadas (universo de interesse - UI) e 99 aplicações de técnicas estatísticas de análise multivariada (população-alvo - PA). 
Tabela 1. Dissertações e teses por ano de defesa

\begin{tabular}{|c|c|c|c|c|c|c|c|c|c|c|c|c|c|c|c|c|c|c|c|c|c|}
\hline \multirow{3}{*}{ ANO } & \multicolumn{9}{|c|}{ FEA/USP } & \multicolumn{9}{|c|}{ EA/UFRGS } & \multirow{2}{*}{\multicolumn{3}{|c|}{ Total }} \\
\hline & \multicolumn{3}{|c|}{ Dissertações } & \multicolumn{3}{|c|}{ Teses } & \multicolumn{3}{|c|}{ Total } & \multicolumn{3}{|c|}{ Dissertações } & \multicolumn{3}{|c|}{ Teses } & \multicolumn{3}{|c|}{ Total } & & & \\
\hline & $\mathrm{U}$ & UI & $\mathrm{PA}$ & $\mathrm{U}$ & UI & PA & $\mathrm{U}$ & UI & $\mathrm{PA}$ & $\mathrm{U}$ & UI & PA & $\mathrm{U}$ & UI & $\mathrm{PA}$ & $\mathrm{U}$ & UI & $\mathrm{PA}$ & $\mathrm{U}$ & UI & PA \\
\hline 2006 & 2 & 0 & 0 & 6 & 5 & 10 & 8 & 5 & 10 & 8 & 2 & 3 & 1 & 0 & 0 & 9 & 2 & 3 & 17 & 7 & 13 \\
\hline 2005 & 12 & 6 & 11 & 3 & 1 & 2 & 15 & 7 & 13 & 4 & 3 & $J$ & 5 & 1 & 2 & 9 & 4 & 7 & 24 & 11 & 20 \\
\hline 2004 & 10 & 5 & 7 & 4 & 2 & 5 & 14 & 7 & 12 & 10 & 4 & 8 & 1 & 0 & 0 & 11 & 4 & 8 & 25 & 11 & 20 \\
\hline 2003 & 9 & 0 & 0 & 3 & 1 & 2 & 12 & 1 & 2 & 9 & 3 & 4 & 0 & 0 & 0 & 9 & 3 & 4 & 21 & 4 & 6 \\
\hline 2002 & 2 & 1 & 4 & 4 & 3 & 5 & 6 & 4 & 9 & 17 & 3 & 6 & 1 & 0 & 0 & 18 & 3 & 6 & 24 & 7 & 15 \\
\hline 2001 & 8 & 1 & 1 & 1 & 1 & 3 & 9 & 2 & 4 & 19 & 5 & 8 & 2 & 1 & 1 & 21 & 6 & 9 & 30 & 8 & 13 \\
\hline 2000 & 12 & 0 & 0 & 5 & 1 & 1 & 17 & 1 & 1 & 11 & 5 & 9 & 0 & 0 & 0 & 11 & 5 & 9 & 28 & 6 & 10 \\
\hline 1999 & 3 & 1 & 1 & 1 & 0 & 0 & 4 & 1 & 1 & 5 & 1 & 1 & 1 & 0 & 0 & 6 & 1 & 1 & 10 & 2 & 2 \\
\hline 1998 & 2 & 0 & 0 & 2 & 0 & 0 & 4 & 0 & 0 & 4 & 0 & 0 & 0 & 0 & 0 & 4 & 0 & 0 & 8 & 10 & 0 \\
\hline 1997 & 5 & 0 & 0 & 0 & 0 & 0 & 5 & 0 & 0 & 3 & 0 & 0 & 1 & 0 & 0 & 4 & 0 & 0 & 9 & 0 & 0 \\
\hline Total & 65 & 14 & 24 & 29 & 14 & 28 & 94 & 28 & 52 & 90 & 26 & 44 & 12 & 2 & 3 & 102 & 28 & 44 & 196 & 56 & 99 \\
\hline
\end{tabular}

$\mathrm{U}$ - Universo

UI - Universo de interesse

PA - População-alvo

\section{Desenho metodológico da pesquisa}

A construção metodológica da pesquisa empírica foi orientada, inicialmente, pela avaliação do processo de solução do problema gerador de dissertações e teses com aplicação de técnica estatística de análise multivariada, na temática aqui selecionada.

Considerou-se, ainda, o fato de que esta avaliação exige ser orientada por um critério, o qual foi construído dentro desse próprio estudo, não tendo sido submetido a um processo de validação anterior.

Essas duas condições, por si só, remetem esse estudo ao âmbito da pesquisa exploratória. Com efeito, o aprofundamento do entendimento do problema foi efetuado com a finalidade de subsidiar a construção de um critério que permitisse avaliar se a aplicação da estatística multivariada aos trabalhos selecionados do período 1997-2006 é adequada. Nesse sentido, este estudo insere-se na abordagem quantitativa, e utiliza a análise de conteúdo.
O delineamento exploratório para este estudo vai ao encontro da abordagem de Selltiz, Malufe e Gatti (1974, p. 60), que evidencia ser uma das finalidades desse método a apresentação de um recenseamento de problemas considerados urgentes por pessoas que trabalham em determinado campo de relações sociais. Exemplifica essa convergência o alerta feito em outros estudos de que a rigidez teórica explicitada nas premissas para aplicações das técnicas estatísticas de análise multivariada não é acompanhada de rigidez empírica explicitada nas concessões feitas pelos pesquisadores. Esse procedimento, por vezes, pode resultar em conclusões não precisas sobre a solução dos problemas.

Aopção pelo uso da técnica de análise de conteúdo foi orientada especialmente pela necessidade primária de esse estudo interpretar a situação problema das dissertações e teses selecionadas, com o propósito de identificar o processo de sua solução por meio da seleção de uma técnica estatística de análise multivariada pertinente. 
Da mesma forma, como foi encontrado na abordagem de vários autores, entre eles Richardson (1999, p. 221-222), também aqui a identificação precisa da natureza da técnica de análise oscila entre a discussão quantitativa e a qualitativa. Assim, de um lado é buscada uma objetividade bastante intensa (RICHARDSON, 1999, p. 221) para categorização dos problemas das dissertações e teses, mediante a definição de critérios rígidos de julgamento da solução desses problemas. De outro, o processo geral de avaliações das unidades de análise (dissertações e teses) está contaminado por julgamentos, pois a análise do conteúdo também supõe o exame das características ausentes ou registros parciais do atendimento às premissas da técnica estatística utilizada.

Nesse sentido, algumas definições de análise de conteúdo parecem contemplar o caminho metodológico da coleta de dados, de acordo com propostas encontradas em Janis et al. (apud RICHARDSON, 1999, p. 222): “Assim, a análise de conteúdo pode ser definida como qualquer técnica: na base de regras explicitamente formuladas e sempre quando os juízos do analista sejam considerados como relatórios de um observador científico."

Entretanto, a definição encontrada em Bardin (1979, p. 31) propicia a convivência com as duas abordagens, na medida em que inclui em sua definição de análise de conteúdo, a geração de indicadores quantitativos ou não no processo analítico.

“Análise de conteúdo é um conjunto de técnicas de análise das comunicações, visando obter, através de procedimentos sistemáticos e objetivos de descrição dos conteúdos das mensagens, indicadores (quantitativos ou não), que permitam inferir conhecimentos relativos às condições de produção dessas mensagens." (BARDIN, 1979, p. 31).

A construção do critério de avaliação da aplicação da técnica estatística de análise de regressão logística
Dois critérios são apresentados a seguir com a finalidade de avaliar o grau de acerto na aplicação da análise de regressão logística.

Procedimento de categorização do problema de pesquisa da unidade de análise: Critério 1

Embora várias técnicas de análise multivariada tenham sido utilizadas no material analisado, neste artigo serão salientados os resultados correspondentes à pertinência e adequação do emprego da análise de regressão logística. A orientação para categorizar o problema de pesquisa de cada unidade de análise (unidade "i" de análise) foi dada pela finalidade ou objetivo teórico de aplicação de diferentes técnicas estatísticas de análise multivariada. Segundo Johnson e Wichern (1998, p. 2), as categorias são sintetizadas em cinco modalidades:

- Redução dos dados ou simplificação estrutural.

- Agrupamento.

- Dependência entre variáveis.

- Predição.

- Formulação de hipóteses e testes.

No caso da análise de regressão logística, o objetivo alcançado em seu uso pode ser a investigação da dependência entre variáveis ou predição.

A categoria identificada na unidade "i" de análise foi confrontada com a categoria-objetivo da técnica de análise de regressão logística, na condição de tratamento estatístico aplicado para solução do problema. A avaliação feita por meio desse confronto tem um caráter dicotômico, ou seja, foi considerada como uma aplicação adequada da técnica análise de regressão logística quando a categoria-objetivo de aplicação da técnica ajustouse à categoria do problema de pesquisa da unidade "i” de análise. 
Procedimento de avaliação do atendimento às premissas da técnica estatística de análise de regressão logística: Critério 2

$\mathrm{O}$ atendimento às premissas da análise de regressão logística foi decorrente da confirmação de que todas as premissas postuladas pela teoria foram atendidas. A violação de pelo menos uma das premissas, explicitada no conteúdo apresentado na unidade " $i$ " de análise, bem como a não explicitação da situação de cada premissa (atendimento ou não atendimento) resultou na categoria "não atendimento às premissas". Dessa forma, o critério de avaliação do atendimento às premissas é dicotômico: atendimento a todas as premissas da análise de regressão logística e não atendimento a pelo menos uma das premissas desta técnica.

Avaliação final do nível de adequação do uso da análise de regressão logística

O registro do nível de adequação do uso da análise de regressão logística foi feito em três níveis (Figura 1)

- Nível 1 (Não adequação do uso da técnica): falta de ajuste da categoria-objetivo de aplicação da análise de regressão logística à categoria do problema de pesquisa da unidade “i” de análise, independentemente de serem ou não atendidas as premissas desta técnica (Não atendimento ao critério "1").

- Nível 2: ajuste da categoria-objetivo de aplicação da análise de regressão logística à categoria do problema de pesquisa da unidade "i" de análise e falta de atendimento a pelo menos uma das premissas da técnica ou falta de explicitação da situação de cada premissa (atendimento ou não atendimento), no documento da unidade "i”" (Atendimento ao critério " 1 " e não atendimento ao critério " 2 ").

- Nível 3: ajuste da categoria-objetivo de aplicação da técnica à categoria do problema de pesquisa da unidade " $i$ " de análise e atendimento de todas as premissas da técnica, conforme documento da unidade "i". (Atendimento ao Critério 1 e ao Critério 2).

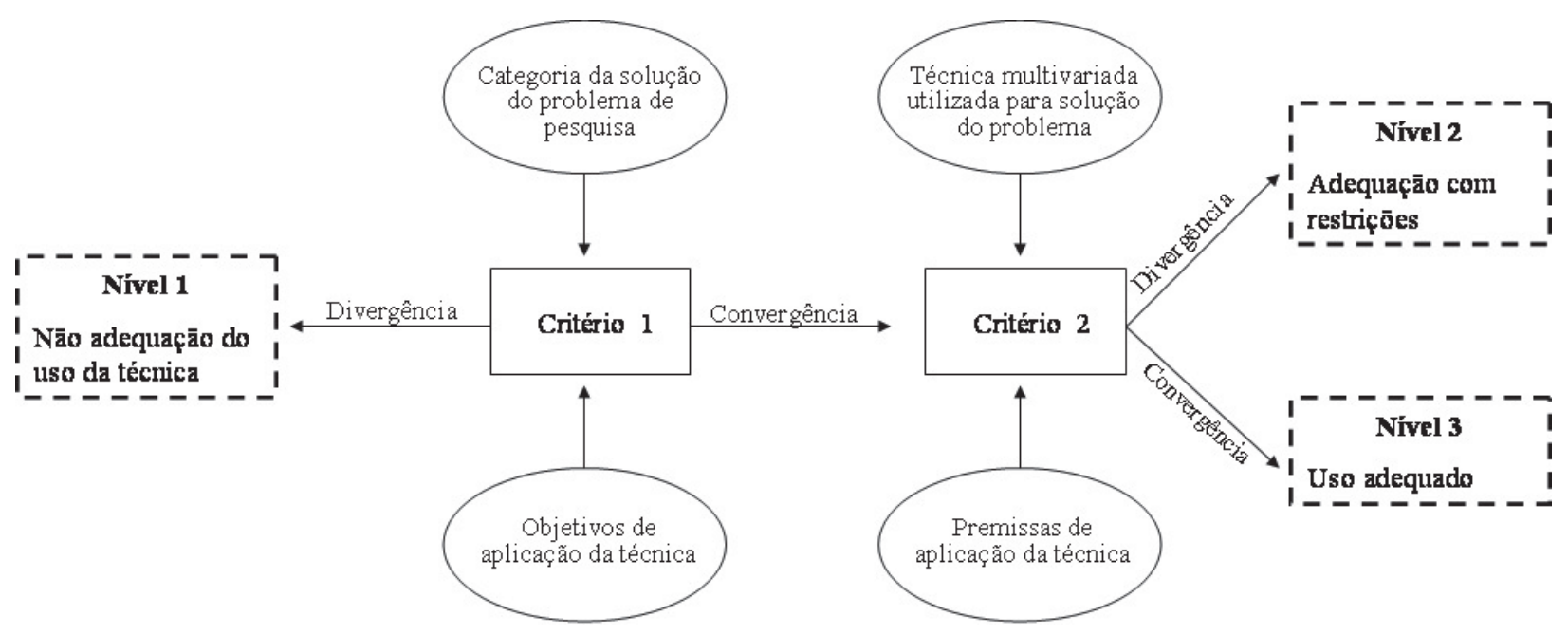

Figura 2. Avaliação do nível de adequação da técnica 


\section{Instrumento de coleta de dados e resultados}

Para a coleta de dados foi desenvolvido um instrumento para registro das ocorrências encontradas em cada unidade " $i$ " de análise.

Esses registros referem-se a:

- Identificação numérica das unidades de análise.

- Ano de defesa do trabalho.

- Descrição do problema de pesquisa.

- Categorização do problema de pesquisa, a partir dos objetivos de aplicação das técnicas de análise multivariada.

- Identificação da técnica de análise multivariada utilizada no trabalho.

- Aplicação do Critério 1: categorização do problema de pesquisa da unidade de análise.

- Identificação e contagem das premissas atendidas na aplicação da análise de regressão logística, se esta foi utilizada.

- Aplicação do Critério 2: procedimento de avaliação do atendimento às premissas da análise de regressão logística.

- Avaliação final do nível de adequação do uso da análise de regressão logística.

Os resultados são identificados por meio da ocorrência evidenciada em cada critério. A partir desses resultados, a complementação do plano analítico pressupõe:

- Evidenciar as técnicas estatísticas de análise multivariada mais utilizadas no período definido para o estudo, independentemente da categoria dos problemas de pesquisa.

- Evidenciar as categorias dos problemas de pesquisa que estariam apresentando maior número de ocorrências de aplicação.

- Evidenciar as premissas mais negligenciadas no processo de aplicação da análise de regressão logística.

\section{Análise dos resultados}

A primeira etapa de análise dos resultados tratou de apresentar as evidências quanto à intensidade de uso das técnicas multivariadas de modo geral, conforme o objetivo "a".

A segunda parte, conforme o objetivo "b", tratou do nível de adequação do uso da análise de regressão logística aos problemas de pesquisa das dissertações e teses sob análise.

A terceira parte identificou, a partir do não atendimento às premissas básicas de aplicação desta técnica, fontes potenciais de erro.

\section{Resultados relativos ao objetivo "a"}

Entre 1997 e 2006, as dissertações e teses acerca da temática Comportamento do Consumidor, defendidas nos PPGA's da FEA/USP e EA/UFRGS, apresentaram maior intensidade de uso de técnicas estatísticas multivariadas para atender ao objetivo de reduzir ou simplificar a estrutura de dados coletada $(62,5 \%)$ com a aplicação da técnica de Análise Fatorial Exploratória, única representante dessa categoria, conforme é apresentado na figura 2 .

A categoria de investigação de dependência entre variáveis foi a segunda mais utilizada (46,4\%). Nesse contexto, seis técnicas de análise multivariada apresentam essa categoria como um dos objetivos de aplicação: Análise de Regressão (15 casos), Análise Discriminante (3 casos), Análise Multivariada da Variância (3 casos), Análise Conjunta (3 casos), Regressão Logística (1 caso) e Correlação Canônica (1 caso).

A Construção de Hipóteses e Testes, exclusivamente representada neste estudo pela técnica estatística multivariada de Modelagem de Equações Estruturais somou 18 casos $(32,1 \%)$.

Já a categoria de agrupamento de objetos ou variáveis foi utilizada em 18 estudos $(33,9 \%)$, e um maior número de casos usou da técnica de 
Análise de Conglomerados (12 casos), seguida da técnica de Análise de Correspondência (5 casos) e da técnica de Escalonamento Multidimensional (1 caso).

Chama a atenção que apenas um dos estudos selecionados utilizou-se de técnica de análise multivariada (regressão logística), com o objetivo de predizer algum fenômeno ou fato. Esse fato possivelmente ocorre mais pela ausência de interesse por problemas de pesquisa na temática de Comportamento do Consumidor do que pela restrição do número de técnicas disponíveis, pois essa categoria é representada, neste estudo, por três técnicas de análise multivariada: Análise de Regressão, Análise Discriminante e Regressão Logística.
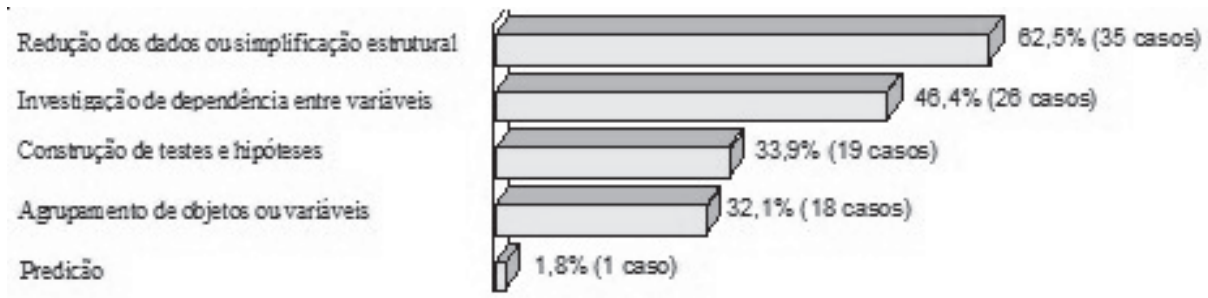

Figura 3. Categoria dos objetivos de aplicação das técnicas estatísticas multivariadas (base: universo de interesse).

Ainda, a fim de ilustrar o ambiente de uso das técnicas estatísticas multivariadas, registre-se que, em $64,3 \%$ dos estudos selecionados, pelo menos duas técnicas são utilizadas para atendimento à solução dos problemas de pesquisa. O uso mais intenso das técnicas ocorre, em termos relativos, nas teses, com $81,2 \%$ dos casos com aplicação de pelo menos duas técnicas, contra 57,5\% nas dissertações, sugerindo maior sofisticação dos estudos no primeiro grupo

Tabela 2. Quantidade de técnicas de análise multivariada diferentes usadas nas dissertações e teses

\begin{tabular}{l|c|c|c}
\hline \hline Técnica estatística de análise multivariada & \multicolumn{2}{|c|}{ Nível do estudo } & Total \\
& Dissertação & Tese & $\begin{array}{c}\text { (dissertações e } \\
\text { teses })\end{array}$ \\
\hline Uma técnica & $42,5 \%(17$ casos $)$ & $18,8 \%(3$ casos $)$ & $35,7 \%(20$ casos $)$ \\
\hline Duas técnicas & $47,5 \%(19$ casos $)$ & $68,7 \%(11$ casos $)$ & $53,6 \%(30$ casos $)$ \\
\hline Três técnicas & $7,5 \%(3$ casos $)$ & $12,5 \%(2$ casos $)$ & $8,9 \%(5$ casos $)$ \\
\hline Quatro técnicas & $2,5 \%(1$ caso $)$ & Nenhum caso & $1,8 \%(1$ caso $)$ \\
\hline Total de casos & $100 \%(40$ casos $)$ & $100 \%(16$ casos $)$ & $100 \%(56$ casos $)$ \\
\hline \hline Média de técnicas & 1,7 & 1,9 & 1,8 \\
\hline Mediana de técnicas & 2,0 & 2,0 & 2,0 \\
\hline Moda de técnicas & 2,0 & 2,0 & 2,0 \\
\hline \hline
\end{tabular}


Quanto às categorias de aplicação da análise de regressão logística ao longo do período 1997-2006, deve-se registrar que a categoria de investigação da dependência entre variáveis apresentou tendência de queda no período 1999-2003, voltando a crescer a partir de 2004, e a categoria predição só apresentou uso em 2004. Nos anos de 1997 e 1998 não se detectou a aplicação de técnica estatística multivariada nas Instituições de ensino alvo desse estudo. A Tabela 3 apresenta a evolução do uso das técnicas estatísticas a partir dos objetivos de investigação da dependência entre variáveis e predição e a distribuição específica do emprego da análise de regressão logística

Tabela 3. Distribuição do uso das técnicas com objetivo de investigação da dependência entre variáveis e predição e uso da análise de regressão logística, ao longo do tempo (período 1997-2006).

\begin{tabular}{l|c|c|c|c|c|c|c|c}
\hline \hline & 1999 & $\mathbf{2 0 0 0}$ & $\mathbf{2 0 0 1}$ & $\mathbf{2 0 0 2}$ & $\mathbf{2 0 0 3}$ & $\mathbf{2 0 0 4}$ & $\mathbf{2 0 0 5}$ & $\mathbf{2 0 0 6}$ \\
\hline $\begin{array}{l}\text { Investigação da } \\
\text { dependência entre variáveis }\end{array}$ & 1 caso & 3 casos & 5 casos & 3 casos & $\begin{array}{l}\text { Nenhum } \\
\text { caso }\end{array}$ & 8 casos & 8 casos & 4 casos \\
\hline Predição & $\begin{array}{l}\text { Nenhum } \\
\text { caso }\end{array}$ & $\begin{array}{l}\text { Nenhum } \\
\text { caso }\end{array}$ & $\begin{array}{l}\text { Nenhum } \\
\text { caso }\end{array}$ & $\begin{array}{l}\text { Nenhum } \\
\text { caso }\end{array}$ & $\begin{array}{l}\text { Nenhum } \\
\text { caso }\end{array}$ & 1 caso & $\begin{array}{c}\text { Nenhum } \\
\text { caso }\end{array}$ & $\begin{array}{c}\text { Nenhum } \\
\text { caso }\end{array}$ \\
\hline $\begin{array}{l}\text { Análise de regressão } \\
\text { logística }\end{array}$ & $\begin{array}{l}\text { Nenhum } \\
\text { caso }\end{array}$ & $\begin{array}{l}\text { Nenhum } \\
\text { caso }\end{array}$ & $\begin{array}{l}\text { Nenhum } \\
\text { caso }\end{array}$ & $\begin{array}{l}\text { Nenhum } \\
\text { caso }\end{array}$ & $\begin{array}{l}\text { Nenhum } \\
\text { caso }\end{array}$ & 1 caso & 1 caso & $\begin{array}{l}\text { Nenhum } \\
\text { caso }\end{array}$ \\
\hline \hline
\end{tabular}

\section{Resultados relativos ao objetivo "b"}

Um resultado positivo deste estudo é a coerência nas dissertações e teses selecionadas quanto à adequação da técnica escolhida de análise de regressão logística ao problema de pesquisa. Assim, pelo critério "1", todas as aplicações da análise de regressão logística são "adequadas".

\section{Resultados relativos ao objetivo "c"}

De modo geral, a análise de regressão logística apresentou razoáveis níveis de atendimento às premissas subjacentes ao seu uso nestas dissertações e teses.

Com uma análise limitada em função do pequeno número de aplicações encontradas nos estudos selecionados, a aplicação foi menos prejudicada até em função do pequeno número de premissas que necessitam ser atendidas (multicolinearidade, tratamento de observações atípicas - outliers e sensibilidade ao tamanho da amostra). Nesses casos analisados, somente o tratamento de observações atípicas (outliers) deixou de ser verificado por uma das aplicações.

Tabela 4 - Atendimento às premissas subjacentes ao uso da análise de regressão logística.

\begin{tabular}{l|c|c}
\hline \hline Premissas subjacentes & $\begin{array}{c}\text { Número de casos } \\
\text { (total de 2 casos) }\end{array}$ & $\begin{array}{c}\text { \% sobre o total de } \\
\text { casos que utilizaram a } \\
\text { técnica }\end{array}$ \\
\hline Multicolinearidade & $\mathbf{1}^{(1)}$ & $100 \%$ \\
\hline Ausência de observações atípicas & 1 & $50 \%$ \\
\hline Tamanho da amostra & 2 & $100 \%$ \\
\hline \hline Atendimento a nenhuma premissa & 0 & $0,0 \%$ \\
\hline Atendimento a até 50\% das premissas & 0 & $0,0 \%$ \\
\hline Atendimento a mais de 50\% das premissas & 1 & $50 \%$ \\
\hline Atendimento a todas as premissas & 1 & $50 \%$ \\
\hline \hline
\end{tabular}

(1) Um dos casos de aplicação da técnica de Regressão Logística tinha como objetivo predição, não exigindo a verificação da multicolinearidade. 
Tabela 5 - Dimensionamentos no geral e segundo a análise de regressão logística

\begin{tabular}{|c|c|c|c|c|c|c|c|}
\hline & \multirow[b]{2}{*}{$\begin{array}{l}\text { Número de } \\
\text { aplicações }\end{array}$} & \multicolumn{2}{|c|}{ Tamanho da amostra } & \multicolumn{2}{|c|}{ Número de variáveis } & \multicolumn{2}{|c|}{$\begin{array}{l}\text { Número de casos } \\
\text { por variável }\end{array}$} \\
\hline & & $\begin{array}{c}\text { Número } \\
\text { médio de } \\
\text { casos }\end{array}$ & $\begin{array}{l}\text { Número } \\
\text { mediano } \\
\text { de casos }\end{array}$ & $\begin{array}{l}\text { Número } \\
\text { médio de } \\
\text { variáveis }\end{array}$ & $\begin{array}{c}\text { Número } \\
\text { mediano } \\
\text { de } \\
\text { variáveis }\end{array}$ & $\begin{array}{c}\text { Número } \\
\text { médio de } \\
\text { casos }\end{array}$ & $\begin{array}{l}\text { Número } \\
\text { mediano } \\
\text { de casos }\end{array}$ \\
\hline Resultado Geral & 99 & 482 & 300 & 23 & 19 & 30 & 14 \\
\hline Análise de regressão logística & 2 & 331 & 331 & 7 & 7 & 51 & 51 \\
\hline
\end{tabular}

\section{Critério "2" de avaliação dos níveis de} adequação da análise de regressão logística

O resultado final da avaliação das aplicações encontradas na população-alvo sugere um frágil ambiente operacional de aplicação da análise de regressão logística, no que se refere ao seu uso e especificamente ao atendimento às premissas subjacentes à técnica.

O emprego do critério " 2 " do processo de avaliação revela que $50 \%$ das aplicações de análise de regressão logística atendem plenamente às suas premissas.

\section{Avaliação final do nível de adequação do uso da análise de regressão logística}

A avaliação final do nível de adequação do uso da análise de regressão logística pode ser sintetizada pelos índices percentuais obtidos nos três níveis retratados na Figura 1:

- Nível 1: Tdoas as aplicações da técnica de análise de regressão logística apresentaram convergência entre a categoria-objetivo de aplicação desta técnica com a categoria do problema da pesquisa;

- Nível 2: Uma das aplicações da análise de regressão logística atendeu parcialmente as suas premissas;

- Nível 3: Uma das aplicações da análise de regressão logística apresentou uso adequado quanto ao atendimento de suas premissas.

\section{Conclusões}

Nos trabalhados avaliados, as soluções para os problemas de pesquisa concentraram-se no emprego de técnicas de redução ou simplificação estrutural dos dados (35s trabalhos), seguido de técnicas de investigação de dependência entre variáveis $(46,4 \%$ dos trabalhos).

No conjunto dos trabalhos focalizados, a análise de regressão logística só passou a ser aplicada em 2004, sendo empregada em trabalho em cada ano.

Um fato bastante positivo quanto à aplicação da análise de regressão logística é que as duas aplicações apresentaram-se adequadas à resolução dos problemas de pesquisa das dissertações e teses focalizadas nesse estudo.

Quanto à qualidade de aplicação desta técnica, cabe realçar que a verificação de todas as suas premissas ocorreu em apenas uma das aplicações.

O foco específico em cada premissa da análise de regressão logística evidencia um maior atendimento à premissa de tamanho da amostra, com verificação nos dois estudos em análise. Cabe mesmo afirmar que esse ambiente de resultados delineia uma intensidade maior de atendimento às premissas que exijam pouco envolvimento do pesquisador quando da aplicação das técnicas. 
Assim, a premissa de tamanho da amostra ocorre intencionalmente no desenho, a priori, da amostra, levando-se em conta apenas padrões de construção de amostras (especificidades do público, tipo de coleta, margem de erro, nível de significância etc).

Todo esse ambiente de evidências revela, a partir da aplicação do critério "2", apenas uma aplicação adequada entre as 2 aplicações de análise de regressão estudadas.

Assim, os achados desse estudo não possam ser extrapolados para a produção acadêmica de outras Instituições ou de outros períodos de tempo, porém eles sugerem a necessidade de maior cuidado conceitual nas aplicações da técnica de análise de regressão logística.

A partir dos resultados aqui obtidos, pode-se ressaltar a importância das bancas de qualificação no sentido de alertar os pesquisadores sobre a necessidade desses cuidados, incentivando a prática do atendimento às premissas subjacentes desta técnica.

Assim, espera-se que o resultado apurado nesse estudo, relativo ao razoável nível de não adequação do uso da análise de regressão logística na populaçãoalvo, em função da não verificação de suas premissas, seja um estímulo aos pesquisadores para a busca de um entendimento mais pormenorizado e aprofundado da técnica a ser empregada como parte do processo de solução do problema de pesquisa, de forma que se minimizem eventuais erros decorrentes da aplicação não adequada.

\section{Referências}

ARKADER, Rebecca. A pesquisa científica em gerência de operações no Brasil. Revista de Administração de Empresas - RAE, São Paulo, v. 43, n. 1, p. 70-79, 2003.

BARDIN, Laurence. Análise de conteúdo. Lisboa: Ed. 70, 1979.

BERTERO, Carlos Osmar; KEINERT, Tânia Margarete Mezzomo. A evolução da análise organizacional no Brasil (1961-93). Revista de Administração de Empresas - RAE, São Paulo, v. 34, n. 3, p. 81-90, 1994.

BERTERO, Carlos Osmar; CALDAS, Miguel Pinto;
WOOD JUNIOR, Thomaz. Produção científica em administração de empresas: provocações, insinuações e contribuições para um debate local. Revista de Administração Contemporânea - RAC, Curitiba, v. 3, n. 1, p. 147-178, jan./abr. 1999.

BOTELHO, Delane; MACERA, Andrea Pereira. Análise meteórica de teses e dissertações da área de marketing apresentadas na FGV-EAESP (1974-1999). In: ENCONTRO ANUAL DA ASSOCIAÇÃO NACIONAL DOS PROGRAMAS DE PÓS-GRADUAÇÃO E PESQUISA EM ADMINISTRAÇÃO, 25., Campinas. Anais... Campinas: ANPAD, 2001. CD-ROM

BREI, Vinícius Andrade; LIBERALI NETO, Guilherme. $\mathrm{O}$ uso de modelagem em equações estruturais na área de marketing no Brasil. In: ENCONTRO DE MARKETING DA ASSOCIAÇÃO NACIONAL DE PROGRAMAS DE PÓS-GRADUAÇÃO EM ADMINISTRAÇÃO EMA, 1., Porto Alegre. Anais... Porto Alegre: ANPAD, 2004. CD-ROM

CALDAS, Miguel Pinto; TONELLI, Maria José; LACOMBE, Beatriz. Espelho, espelho meu: metaestudo da produção científica em recursos humanos nos ENANPADs da década de 90. In: ENCONTRO DA ASSOCIAÇÃO NACIONAL DE PROGRAMAS DE PÓS-GRADUAÇÃO EM ADMINISTRAÇÃO EnANPAD, 26., Salvador. Anais... Salvador: ANPAD, 2002. CD-ROM

CRAMER, Jan Salomon. The origins of logistic regression. Amsterdam: Tinbergen Institute, 2002.

FREES, Edward. H. Data analysis using regression models: the business perspective. New Jersey: Prentice Hall, 1996.

GAMBOA, Silvio Ancízar Sánchez. Epistemologia da pesquisa em educação. 1987. Tese (Doutorado em Filosofia e Historia da Educação) - Unicamp, Campinas.

GARSON, G. David. Testing of assumption, from Statnotes: topics im multivariate analysis. 2007a. Disponivel em: <http://www2.chass.ncsu.edu/garson/ pa765.statnote.html>. Acesso em: 20 jul. 2007.

Logistic regression, from Statnotes: topics im multivariate analysis. 2007c. Disponível em: <http:// www2.chass.ncsu.edu/garson/pa765.statnote.html>. Acesso em: 20 jul. 2007.

GUJARATI, Damodar N. Econometria básica. São Paulo: Makron Books, 2000.

HAIR, Joseph. F.; TATHAM, Ronald L.; ANDERSON, Rolph E.; BLACK, Willian C. Análise multivariada de dados. 5. ed. Porto Alegre: Bookman, 2005.

HOPPEN, Norberto; AUDY, Jorge Luiz Nicolas; 
ZANELA, Amarolinda Iara da Costa; CANDOTTI, Clarissa Tarrago; SANTOS, André Moraes; SCHEID, Roberto; PERIN, Marcelo Gattermann; MECCA, Marlei Salete; PETRINI, Maira. Sistemas de informação no Brasil: uma análise dos artigos científicos dos anos 90 . In: ENCONTRO NACIONAL DOS PROGRAMAS DE PÓS-GRADUAÇÃO EM ADMINISTRAÇÃO EnANPAD, 22., 1998, Foz do Iguaçu. Anais... Foz do Iguaçu: ANPAD, 1998. p. 36.

JOHNSON, Richard A.; WICHERN, Dean W. Applied multivariate statistical analysis. 4. ed. New Jersey: Printice Hall, 1998.

LEAL, Ricardo Pereira Câmara; OLIVEIRA, Jefferson; SOLURI, Aline Feldman. Perfil da pesquisa em finanças no Brasil. Revista de Administração de Empresas, São Paulo, v. 43, n. 1, p. 91-103, 2003.

LOURENÇO, Alexandre.; MATIAS, Rui. P. Estatística multivariada. Porto: Instituto Superior de Engenharia do Porto, 2001.

MACHADO- DA- SILVA, Clóvis Luiz; CUNHA, Vera C.; AMBONI, Nério. Produção acadêmica em administração pública: período 1983-1988. In: ENCONTRO ANUAL DA ASSOCIAÇÃO NACIONAL DE PROGRAMAS DE PÓS-GRADUAÇÃO E PESQUISA EM ADMINISTRAÇÃO, Belo Horizonte: ANPAD, 1989, p. 1599-1618.

Organizações: o estado da arte da produção acadêmica no Brasil. In: ENCONTRO NACIONAL DA ASSOCIAÇÃO NACIONAL DOS PROGRAMAS DE PÓS-GRADUAÇÃO E PESQUISA EM ADMINISTRAÇÃO, 14., 1990, Belo Horizonte. Anais... Belo Horizonte: ANPAD, 1990. p. 11-28.

MALHOTRA, Naresh K. Pesquisa de marketing. Porto Alegre: Bookman, 2001.

MARTINS, Gilberto de Andrade. Epistemologia da pesquisa em administração. 1994. Tese (Livre-Docência. Faculdade de Administração, Economia e Contabilidade) - Universidade de São Paulo, São Paulo.

MILAGRE, Rosileia A. Estatística: uma proposta de ensino para os cursos de administração de empresas. 2001. Dissertação (Mestrado em Engenharia de Produção) - Universidade Federal de Santa Catarina, Florianópolis, 2001.

PERIN, Marcelo Gattermann; SAMPAIO, Claudio Hoffmann; FROEMMING, Lurdes Marlene Seide; LUCE, Fernando Bins. A pesquisa Survey em artigos de Marketing nos ENANPADs da Década de 90. Revista Interdisciplinar de Marketing, Maringá, v. 1, n. 1, p. 4459, jan./abr. 2002.

PEDUZZI, Peter; CONCATO, John; KEMPER,
Elizabeth; HOLFORD, Theodore R.; FEINSTEIN, Alvan R. A simulation of the number of events per variable in logistic regression analysis. Journal of Climical Epidemiology, v. 49, n. 12, p. 1373-1379, Dec.1996.

PESTANA, Maria Helena; GAGEIRO, João Nunes. Análise de dados para ciências sociais: a complementaridade do SPSS. 2. ed. Lisboa: Silabo, 2000.

POWERS, Thomas; SWAN, John E.; BOS, Theodore; PATTON, John Frank. Carrer research productivity patterns of marketing academicians. Journal of Business Research, New York, v. 42, n. 1, p. 75-86, 1998.

RICHARDSON, Roberto Jerry. Pesquisa social: métodos e técnicas. 3. ed. São Paulo: Atlas, 1999.

RODRIGUES FILHO, José. Estudos críticos em administração no Brasil: Classificação da produção de conhecimento sob a ótica da teoria crítica de Jurgen Habermas. 2002. Tese - Universidade Federal da Paraíba, Paraiba.

ROESCH, Sylvia Maria Azevedo; ANTUNES, Elaine Di Diego; SILVA; Lisiane Vasconcellos da. Tendências da Pesquisa em recursos humanos e organizações: uma análise das dissertações de mestrado. In: ENCONTRO DA ASSOCIAÇÃO NACIONAL DE PROGRAMAS DE PÓS-GRADUAÇÃO EM ADMINISTRAÇÃO ENANPAD, 22., 1997, Rio de Janeiro. Anais... Rio das Pedras: ANPAD, 1997.

SELLTIZ, Claire; MALUFE, Jose Roberto; GATTI, Bernardete A. Métodos de pesquisa nas relações sociais. São Paulo: Herder, 1974.

STEINER, Maria Teresinha Arns. Uma metodologia para o reconhecimento de padrões multivariados com resposta dicotômica. 1995. Tese (Doutorado em Engenharia) - Universidade Federal de Santa Catarina, Florianópolis. Disponível em: <http://www.eps.ufsc.br/ teses/steiner/capit_2/cap2_ste.htm>. Acesso em: 28 out. 2007.

TABACHNICK, Barbara G.; FIDELL, Linda S. Using multivariate statistics. 3. ed. New York: Harper Collins, 1996.

TONELLI, Maria José; CALDAS, Miguel P.; LACOMBE, Beatriz Maria Braga, TINOCO, Tatiana. Produção acadêmica em recursos humanos no Brasil: 1991-2000. Revista de Administração de Empresas RAE, São Paulo, v. 43, n. 1, p. 105-122, 2003.

TORRES, Rosane Rivera. Estudo sobre os planos amostrais das dissertações e teses em administração da faculdade de economia, administração e contabilidade da universidade de São Paulo e da escola de administração da universidade Federal do Rio Grande do Sul: uma contribuição crítica. 2000. Dissertação (Mestrado) - 
FEA/USP, São Paulo.

TRIVELLONI, Carlos A. P.; HOCHHEIM, Norbeto. Avaliação de Imóveis com técnicas de análise multivariada. In: CONGRESSO BRASILEIRO DE CADASTRO TÉCNICO MULTIFINALITÁRIO, 1998, Florianópolis, UFSC.

VERGARA, Sylvia Constant; CARVALHO JUNIOR, D. S. Nacionalidade dos autores referenciados na literatura brasileira sobre organizações. In: ASSOCIAÇÃO NACIONAL DE PÓS-GRADUAÇÃO E PESQUISA EM ADMINISTRAÇÃO - ANPAD, 19., 1995, João Pessoa. Anais... João Pessoa: ANPAD, 1995. CD-ROM

VIEIRA, Francisco G. D. Por quem os sinos dobram? uma análise da publicação científica na área de marketing do ENANPAD. In: ENCONTRO NACIONAL DOS PROGRAMAS DE PÓS-GRADUAÇÃO EM ADMINISTRAÇÃO - EnANPAD, 22., 1998, Foz do Iguaçu. Anais... Foz do Iguaçu: ANPAD, 1998. p. 113. 